\title{
Contribución al conocimiento de los Speophilus del grupo kiesenwetteri Diek
}

\author{
Por Oleguer Escolà i Boada ${ }^{1}$ )
}

Col Lamina 42(1) y 1 Figura

\section{Introducción}

Como resultado de una serie de prospecciones sistemáticas por la región de Sant Salvador de les Espases (Esparraguera, Barcelona) el dia 24 de diciembre de 1961 varios miembros del E.R.E. (Equip de Recerques Espeleològiques) del "Centre Excursionista de Catalunya" descubrieron y exploraron una pequeña sima de 8 metros de profundidad a la que dieron el nombre de "Avenc Montserrat".

Por darse la circunstancia de que no participó en dicha exploración ningún biospeleólogo, no se recogió fauna alguna en la citada cavidad.

Ya anteriormente a esta exploración, Don Oscar Andrés, espeleólogo del G.E.S. (del Club Montañés Barcelonés) y excelente amigo nuestro, nos había comunicado su interés por la región de Sant Salvador, en la que creía que, correspondiendo a la continuidad geológica de esta zona respecto a la Sierra de Rubió (en la que se halla enclavada la Cueva del Patracó) podría hallarse, al igual que en la citada localidad, de la que está separada unicamente por el Río Llobregat, el Speophilus patracoi Zar.

Al conocer el descubrimiento del Avenc Montserrat, O. Andrés proyectó una visita a dicha sima, que aplazada por diversas circunstancias no se llegó a efectuar hasta el día 1 de Enero de 1962. En ella logró capturar dos ejemplares de Speophilus, que llevó al Museo de Zoología del que siempre ha sido un activo colaborador.

Dada la escasez del material disponible, optamos, antes de proceder al detenido examen del mismo, por recoger nuevos ejemplares, como así pudo conseguirse mediante sucesivas visitas a la referida cavidad, alternando la recolección directa con el empleo de cebos. Al final de tales exploraciones, llevadas a cabo durante los días 30-XII-62 y 4-I-63, disponíamos de unos veinte ejemplares, número que se juzgó suficiente para el estudio definitivo de los mismos. Observados super-

1) Rafael Batlle 14, Barcelona 17. 
ficialmente dichos ejemplares, evidenciaban sensibles diferencias frente a $S$.patracoi y a las formas hasta hoy conocidas de S.kiesenwetteri hasta el punto de justificar sobradamente el establecimiento para ellos de una nueva raza que debía subordinarse no a $S$.patracoi sino a $S$.kiesenwetteri (2) por ser las formas castellsaperai y san-llorensi de este último a las que más se parecen.

$\mathrm{Y}$ al paso que describimos esta nueva raza creemos conveniente acompañar un breve comentario de su relación con las dos especies de Speophilus que colonizan los macizos vecinos de Montserrat y Sant Llorenç del Munt - Serra de l'Obac y la parte colindante a éstos de la cordillera prelitoral interna; previo un esbozo geográfico de estos relieves.

No podemos iniciar este trabajo sin antes agradecer sinceramente la ayuda que en todo momento hemos recibido de nuestro maestro, el Dr. Francisco Español, sin la cual nos hubiera sido imposible la consecución de esta nota.

\section{Noticia geográfico-geológica de la Zona estudiada}

Separados dos a dos por el río Llobregat se encuentran cuatro macizos calcáreos colonizados cada uno de ellos por una especie o raza diferente de Speophilus.

Geográficamente son dos alineaciones montañosas orientadas de W a $\mathrm{E}$ divididas de $\mathrm{N}$ a $\mathrm{S}$ por el río Llobregat en cuatro zonas morfológicamente distintas.

La alineación septentrional pertenece estructuralmente a la Depresión del Ebro y está formada por conglomerados "supranumulíticos" cuya resistencia a la erosión es responsable de las alturas actualmente alcanzadas: al $\mathrm{W}$ del Llobregat el conocido macizo de Montserrat (1224 m.) y al E las sierras de Sant Llorenç del Munt y Serra de l'Obac que alcanzan los $1095 \mathrm{~m}$. Morfológicamente se ha convenido en calificar estos relieves como integrantes de la Cordillera Prelitoral Externa (10).

Por su parte los escasos relieves meridionales, a pesar de su pobre desarrollo, integran en sentido estructural la verdadera Cordillera Prelitoral. Pero mientras la parte W - formada por la Sierra de Rubió queda prácticamente anulada con sus $430 \mathrm{~m}$. de altura a los pies de Montserrat, con cerca de $1300 \mathrm{~m}$., la zona E situada frente a la ancha depresión existente entre Montserrat y St. Llorenç del Munt se eleva unos $300 \mathrm{~m}$. por encima de ella a pesar de que sus cotas máximas no llegan a alcanzar los $650 \mathrm{~m}$. A diferencia de la zona de conglomerados de la Depresión del Ebro esta cordillera prelitoral interna está formada 
por materiales mesozóicos : calizas y areniscas del Muschelkalk y Buntsandstein que reposan sobre el zócalo silúrico.

Así pues un análisis de cada uno de los macizos señalados podría resumirse de la siguiente manera:

A. Montserrat. Bloque uniforme perfectamente delimitado, integrado exclusivamente por conglomerados con numerosas intercalaciones de margas y areniscas de escasa potencia que no afectan de modo sustancial la evolución del conjunto. El macizo surge bruscamente del llano de la Depresión por la parte de Manresa, así como de Igualada y del Bruc, y está perfectamente delimitado al E por el Llobregat y al S por la sierra de Rubió y Collbató.

B. St. Llorenç del Munt - Serra de l'Obac. Relieves situados hacia el este de Montserrat, separados de ella por la depresión de Vacarisses, de unos $10 \mathrm{~km}$. de anchura y de la que es responsable el establicimiento del Llobregat entre ambos macizos.

Integrados por los mismos conglomerados de Montserrat, se hallan formados por dos cadenas paralelas orientadas de $\mathrm{N}$ a $\mathrm{S}$ y separadas por la Riera de les Arenes: St. Llorenç del Munt al E y la Serra de l'Obac al W. Hay entre ellas un claro punto de unión en el Coll de Estenalles, cerca de La Mata.

El contacto con los materiales del interior de la Depresión del Ebro se realiza mediante los bruscos escalones de la parte N. Hacia el E se prolongan algunos relieves por el Turó del Muronell hasta el río Ripoll y por el W el contacto entre la Serra de l'Obac y la depresión de Vacarisses es más suave, verificándose a través de una serie de relieves que van desde Puig Andreu y Turó del Mal Pas hasta el Paller de Tot L'Any.

C. Serra de Rubió ó de La Salut. Pequeña escama de calizas del Muschelkalk que se extiende al S de Montserrat, desde Collbató hasta el Llobregat, en el punto en donde está enclavado el balneario de La Puda de Montserrat. Su máxima altura es de $438 \mathrm{~m}$. Se separa de montserrat por un bajo collado en las proximidades de Collbató, y limita al S con el silúrico mientras que queda disecada al E por una angosta garganta en cuyo fondo se ha encajado el Llobregat.

D. St. Salvador de les Espases. Zona de gran complejidad estructural. Se extiende al E del Llobregat, a continuación de la Serra de Rubió, desde éste hasta la Riera de St. Jaume, que aisla claramente esta parte de la Cordillera Prelitoral Externa. La máxima altura sobrepasa apenas los 500 m. en las Roques De l'Afrau. La gran riqueza tectónica de esta pequeña zona es debida sobre todo al contacto que tiene lugar entre los materiales mesozóicos de la Cordillera Prelitoral - estructuralmente considerada - y la Depresión del Ebro cuyos 
materiales llegan a formar aquí algunos relieves que igualan y aún sobrepasan ligeramente la altura alcanzada en las Roques de l'Afrau.

Los contactos geológicos entre las cuatro zonas estudiadas

Para la interpretación del ciclo evolutivo que ha sufrido el grupo de Speophilus de esta región, es absolutamente necesario tener en cuenta la geología y morfología de los relieves colonizados por estos coleópteros, ya que el tránsito de unas zonas a otras viene rigurosamente condicionado por la existencia de fisuras en el subsuelo que les permitan el paso sin salir a la superficie. Así una separación de dos regiones kársticas debida a una capa de cierto espesor de materiales no karstificables, entraña un aislamiento de los troglobios que puedan colonizar estas dos zonas mientras que un contacto directo entre dos tipos de materiales karstificados no acostumbra a significar separación de ninguna especie. El aislamiento debido a una capa impermeable depende naturalmente de su espesor, de su posible diaclasación y de sus soluciones de continuidad. Un material muy plástico, debido a su escasa diaclasación, ofrecerá mayor separación que otro de mayor rigidez que haya sufrido los mismos esfuerzos tectónicos a lo largo del tiempo.

Debe tenerse en cuenta también la posible formación de cavidades tectónicas en zonas no kársticas, que podrían comportarse en el aspecto biológico de modo análogo a una caverna normal.

\section{A. Contacto entre los dos macizos de conglomerados}

Montserrat y St. Llorenç del Munt se hallan separados geográficamente por el río Llobregat, que discurre a los pies de Montserrat, y por la depresión de Vacarisses, de unos $10 \mathrm{~km}$. de anchura.

También es clara la separación geológica pues mientras los dos macizos están formados por los conglomerados supranumulíticos, la depresión la constituyen el paleoceno y facies rojas continentales. Las pudingas paleocenas, que forman gran parte de la depresión son algo karstificables, pero debido a que no se conoce ninguna cavidad enclavada en ellas, se precisaría un estudio espeleológico de la zona para llegar a alguna conclusión.

\section{B. Contacto entre las dos zonas calizas}

Aparentemente se hallan separadas unicamente por el curso del río Llobregat que diseca las calizas con un desnivel de $450 \mathrm{~m}$. con respecto a las cumbres de ambos lados. Geográficamente éste es el único accidente que se presenta en ellas. Y aunque su valor es notable, pues el 
lecho del río llega casi hasta la base de las calizas en este punto, no es el único factor de aislamiento: al menos en dos puntos, uno a cada lado del Llobregat, afloran los potentes estratos de areniscas del Buntsandstein, que aislan las dos áreas descritas.

\section{El contacto Montserrat - Serra de Rubió}

Los conglomerados de Montserrat no se hallan en contacto directo con el triásico sino que se interponen entre ellos las capas del Paleoceno-Luteciense, que acusan una notable discordancia progresiva en su contacto con el Buntsandstein. Sobre este último se hallan, muy plegadas, las calizas del Muschelkalk. El aislamiento es pues acusado, debido al Paleoceno-Luteciense y al Buntsandstein.

\section{Contacto Serra de l'Obac-St. Salvador}

La separación es aquí perfecta, ya que aparece el sustrato de pizarras silúricas y actúa de formidable barrera entre las dos zonas, cuyos extremos más cercanos se hallan a unos $3 \mathrm{~km}$. uno del otro.

\section{Estudio faunístico}

\section{Resumen histórico}

En 1869 el entomólogo alemán Dieck describió de las conocidas cuevas del Salitre de Collbató (Montserrat) el primer coleóptero, cavernícola de Cataluña: Perrinia kiesenwetteri (7). Posteriormente Jeannel, en su Monografía de los Bathysciinae (2) dió al género el nombre definitivo de Speophilus.

Algunos años más tarde, en 1924, las prospecciones del Dr. R. Zariquiey Alvarez en la región de St. Llorenç del Munt le llevaron a descubrir numerosos ejemplares de Speophilus ligeramente distintos de la forma tipo, lo que le indujo a describir dos razas geográficas del Speophilus kiesenwetteri: la ssp. san-Llorensi de la Cova de Santa Agnès en la sierra de St. Llorenç, y la var. castellsaperai del Avenc de Castellsapera, en la Serra de l'Obac.

Al mismo tiempo y como consecuencia de una serie de exploraciones, se extendió el área geográfica del S.kiesenwetteri s. str. a todo el macizo de Montserrat. Ulteriores exploraciones ampliaron notablemente la extensión de todas las variedades citadas por todo el relieve de St. Llorenç y Serra de l'Obac.

Dos años antes ya había descubierto el Dr. Zariquiey en la Cueva del Patracó otro coleóptero que describió como una subespecie de $S$.kiesenwetteri: la ssp. patracoi. No obstante, enviado por él mismo al Dr. 
Jeannel para que le diera su opinión sobre la forma descubierta este último creyó necesario considerarla como una especie aparte, criterio que fue aceptado (2) (8).

Las numerosas exploraciones efectuadas hasta hoy en esta región no habían hecho sino confirmar la presencia en todas las cavidades de cada zona del Speophilus correspondiente, aumentando la extensión geográfica de cada forma excepto del $S$.patracoi que continúa hoy día confinado a la citada Cueva del Patracó.

La población de Montserrat reveló ser de gran uniformidad, y por lo que respecta al macizo de St. Llorenç - Serra de l'Obac se han sucedido los hallazgos de un gran número de ejemplares intermedios entre las formas san-llorensi y castellsaperai, hasta el punto de ser hoy día prácticamente inseparables por la morfología, como ha puesto ya de manifiesto Español (3) y ha corroborado Lagar (6). Probablemente estas dos formas corresponden a los tipos extremos de un Rassenkreis, pero antes de sentar una conclusión definitiva a este respecto es preferible resolver de una vez esta cuestión con un estudio exhaustivo apoyado en un análisis biométrico, que dejamos para otra ocasión.

El reciente descubrimiento del nuevo Speophilus, objeto de esta nota, ha venido a completar un paso en la evolución del grupo.

\section{Descripción de la nueva forma}

Speophilus kiesenwetteri ssp. andresi nov.

Morfología de S.kiesenxetteri ssp. san-llorensi del que se diferencia por la talla sensiblemente mayor. Cuerpo más robusto. Pubescencia de la parte dorsal de los élitros más desarrollada. Quilla mesosternal formando un ángulo netamente obtuso frente a la ssp. san-llorensi que la presenta formando un ángulo recto.

De kiesenwetteri s. str. se separa por todos los caracteres descritos y especialmente por la talla todavía mayor.

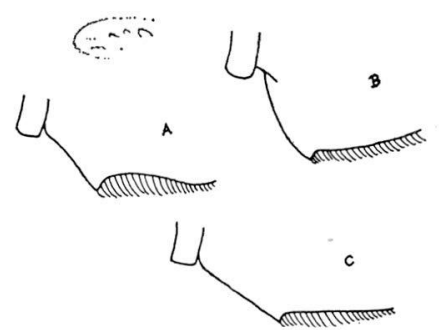

Fig. 1. A. Quilla mesosternal de Speophilus kiesenspetteri ssp. andresi nov. B. Quilla mesosternal de Speophilus kiesenspetteri var. castellsaperai Zar. C. Quilla mesosternal de Speophilus patracoi Zar. 


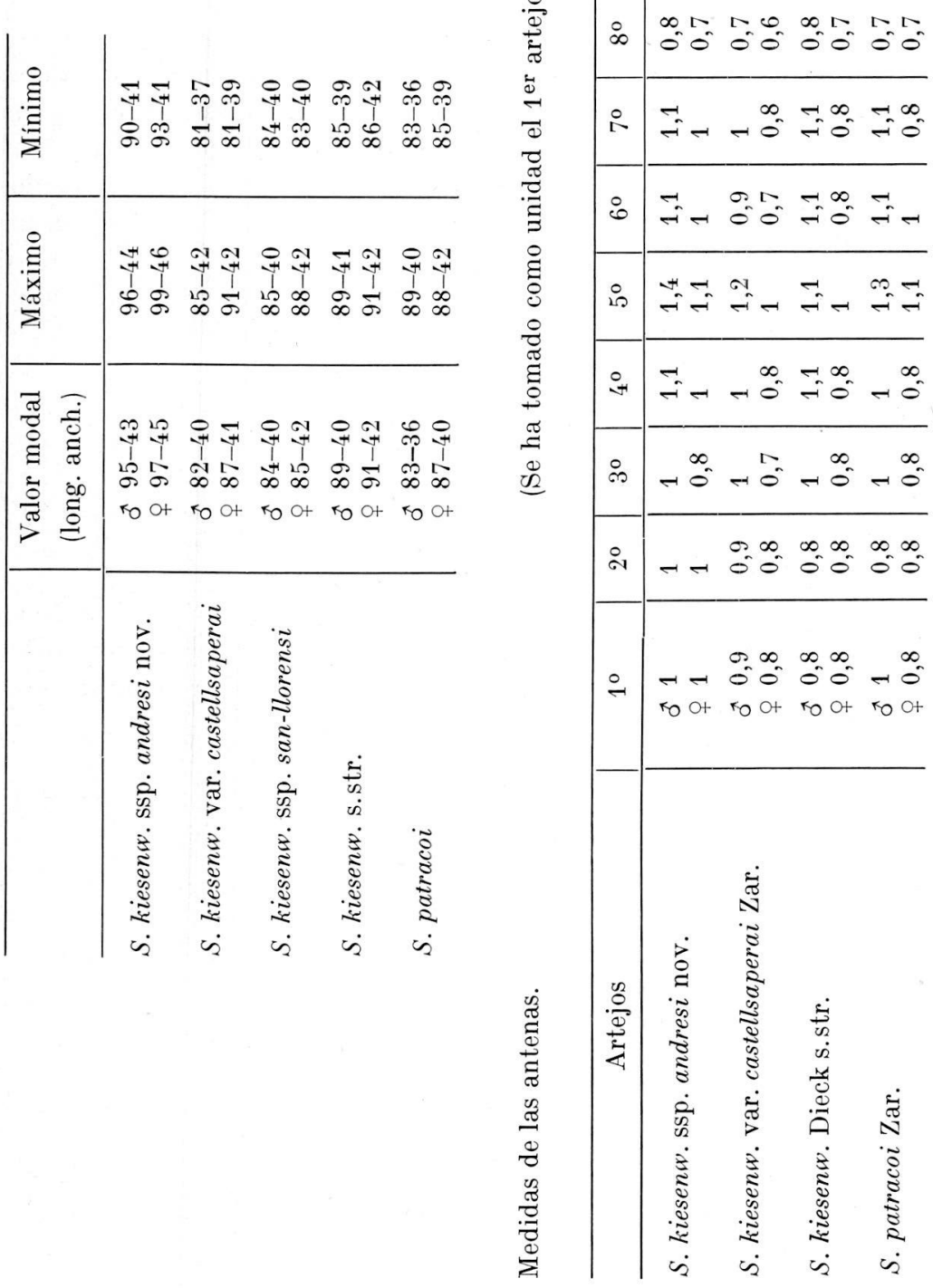

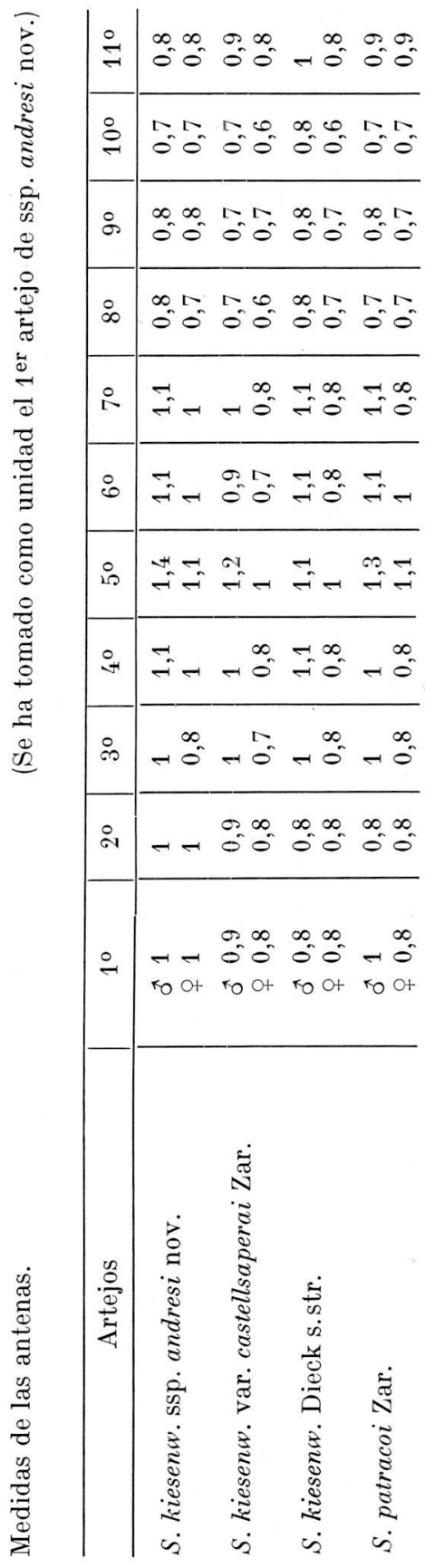


Distinto por otra parte de S.patracoi, del que imita la pubescencia, por la quilla no tan obtusa como en éste y por el contorno diferente del cuerpo.

Dedico esta forma a su descubridor, Don Oscar Andrés, que tuvo a bien llevar los ejemplares recogidos por primera vez al Museo para su estudio.

Biometría. Muy interesante es la biometría del grupo, que presta nuevos elementos de diferenciación entre las distintas formas. Insertamos a continuación un cuadro de valores modales y valores extremos de la longitud y anchura del cuerpo en las diferentes formas. Las medidas han sido tomadas sobre unos 10 a 15 ejemplares de cada sexo y especie, por término medio, escogidos totalmente al azar. Los valores modales pueden tomarse practicamente como valores medios puesto que coinciden con ellos en la mayoría de los casos o difieren muy poco.

Las cifras corresponden a las divisiones de la escala del micrómetro ocular de modo que 28 divisiones equivalen a $1 \mathrm{~mm}$.

\section{Biogeografía y habitat}

Por el momento esta nueva subespecie se halla localizada exclusivamente en la pequeña sima de $8 \mathrm{~m}$. de profundidad llamada "Avenc Montserrat". Esta se halla a $420 \mathrm{~m}$. de altura, en las proximidades de Coll Cassot, no muy lejos de la conocida ermita de St. Salvador.

Por los alrededores de la sima se encuentran muchas otras cavidades de escaso desarrollo, la mayor de las cuales, el Avenc del Clast, tiene sólo $18 \mathrm{~m}$. de profundidad. Pero en ninguna de ellas ha sido posible encontrar ningún ejemplar de Speophilus. Esto no puede ser debido a un aislamiento de las demás cavidades respecto al Avenc Montserrat (se hallan todas ellas en una zona intensamente fracturada y a pocos metros unas de otras) sino que debe atribuírse a la falta de recursos nutricios en las otras cavidades. Con toda probabilidad, colocando cebos en alguna de las simas que reúnen un mínimo de condiciones de humedad, acabarían por acudir los Speophilus de la población que debe colonizar la totalidad de esta reducida zona.

El origen del Avenc Montserrat, al igual que el de las demás simas cercanas, hay que buscarlo en el deslizamiento de la capa de calizas en que se encuentran, sobre las areniscas plásticas del Buntsandstein que les sirven de base, debido al vacío excavado por el Torrente de St. Salvador que circula actualmente a un nivel inferior. No son pues cavidades típicamente kársticas sino verdaderas "grietas de desprendi- 
miento" (11) debidas a este movimiento de la caliza, y probalemente predeterminadas por causas tectónicas.

Pero esta diferencia de origen respecto a las simas típicas no parece tener efecto alguno sobre las poblaciones troglobias mientras existan biotopos adecuados a su desarrollo.

La boca del Avene Montserrat inicia un breve salto de dos metros que da acceso a una rampa, por la que, tras un corto recorrido, se llega a la pequeña sala final de unos $4 \mathrm{~m}$. de diámetro.

El corredor de entrada ofrece sólo condiciones para la vida troglóxena y troglófila. Se ha recogido en esta parte arañas (gen. Meta) e isópodos troglóxenos. En cambio la pequeña sala final reúne buenas condiciones para la vida troglobia: humedad $96 \%$, temperatura $14^{\circ} \mathrm{C}$, una débil corriente de aire hacia el exterior (4-I-63). Por la escasa concreción existente gotea el agua que mantiene la elevada humedad. La sustancia orgánica nutricia procede de detritus vegetales arrastrados por el agua a través de las grietas del techo, del que cuelgan algunas raíces, y esencialmente de pequeñas manchas de guano de murciélagos aislados que se refugian temporalmente en la cueva. Sólo se han observado durante las visitas efectuadas dos murciélagos de herradura de la especie Rhinolophus hipposideros.

En esta sala, corriendo por entre las piedras que cubren el suelo, y por la concreción húmeda, se encuentran, relativamente abundantes los Speophilus. Se han hallado también en este punto varios isópodos muy despigmentados, campodéidos etc., material que no ha sido todavía estudiado.

\section{Esquema filogenético del grupo kiesenwetteri}

Se conocen pues dos especies de este grupo al W del Llobregat: Speophilus kiesenwetteri Dieck s. str. y Speophilus patracoi Zar., y dos subespecies al otro lado del río: ssp. san-llorensi Zar. (prácticamente inseparable de la var. castellsaperai) y la ssp. andresi nov. Ya el Dr. Zariquiey había propuesto una acertada teoría sobre la evolución de este grupo que suponía un nucleo primitivo, que en su desplazamiento hacia el W. dió la var. castellsaperai, y ésta, al extenderse hacia Montserrat y luego quedar aislada por el Llobregat originó el Speophilus kiesenwetteri s.str., forma que, por un supuesto contacto entre el conglomerado de Montserrat y las calizas de la Serra de Rubió pudo llegar a colonizar esta última zona y por aislamiento posterior diferenciarse el S.patracoi. 
El descubrimiento de la nueva ssp. andresi ha venido a modificar esta teoria sugeriendo una nueva explicación que se adapta mejor a la sistemática del grupo.

En efecto, debido a un contacto directo de los materiales de la Serra de l'Obac y la faja de calizas del Muschelkalk, que se extiende al S., la var. castellsaperai pudo colonizar estas calizas y extenderse por ellas hasta Sant Salvador de les Éspases y la Serra de Rubió. Un aislamiento tectónico entre la Serra de l'Obac y esta zona la aisla del primitivo núcleo, y el Llobregat la subdividió en dos regiones en las que se originaron: en una la ssp., andresi y en la otra, por una diferenciación mayor, el S.patracoi.

Paralelemente a esta rama, la var. castellsaperai, se extiende por el norte por la zona de conglomerados hasta que la disección del Llobregat la divide en dos partes, en la más occidental de las cuales se forma el S.kiesenwetteri s. str.

Un estudio comparativo del conjunto pone de manifiesto, como era de esperar, que las dos formas más extendidas (kiesenwett. s. str. y sanllorensi) responden a las poblaciones menos evolucionadas; mientras que $S$.kiesenw., ssp. andresi y $S$.patracoi de área muy reducida, presentan, como es norma en pequeñas poblaciones aisladas, un grado de evolucion sensiblemente mayor.

El siguiente esquema resume la filogenia del grupo:

CO MGLOMERADOS

Speoph. kiesw. ssp. san-llorensi Zar.

(Sant Llorenç del Munt)

S. kiesw. var. castellsaperai Zar.

(Serra de l'Obac)

S. kiesw. Jeann. s. str.

(Montserrat)
CALIZAS

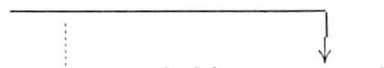

S. kiesw. ssp. andresi nov. (Sant Salvador de les Espases)

S. patracoi $\stackrel{\downarrow}{\text { (Zar.) Jeann. }}$

(Serra de Rubió)

\section{RÉSUMÉ}

L'auteur étudie le groupe kiesenwetteri du genre Speophilus (Col. Catopidae) endémique de la Catalogne (Espagne).

Le travail comprend une analyse géographique et géologique des massifs colonisés par le groupe, avec une étude sur l'isolement géologique des espèces, et l'étude faunistique des 5 formes connues actuellement, l'une d'elles nouvelle. 


\section{RESUMEN}

El autor estudia el, grupo kiesenswetteri del género Spéophilus (Col. Catopidae) endémico de Cataluña (España).

El trabajo comprende un análisis geográfico-geológico de los macizos colonizados por el grupo, junto con un estudio sobre el aislamiento geológico de las especies y el estudio faunístico de las 5 formas conocidas actualmente, una de ellas nueva para la ciencia.

\section{B I B LI OGRAF IA}

1. Jeannel, R. (1911) - Révision des Bathysciinae. Arch.Zool. Exp.étGén., 5 Sér., T. VII, p. 365, pl. I, fig. 31, X fig. 291-294.

2. - (1924) - Monographie des Bathysciinae. Arch. Zool. Exp. ét Gén., T. 63 , fasc. 1, p. 171-172, fig. 185 .

3. Español, F. (1956) - Los Bathysciitae catalanes. $2^{\circ}$ Congr. Int. Est. Pir. 1954, T. 3, pp. 109-123. Toulouse.

4. - (1926) - Contribució al coneixement dels coleópters cavernícoles del massís de St. Llorenç del Munt. Arch. Centr. Exc. Terrassa, Any XVI. $2^{\text {a }}$ época, no 91 , pp. 70-74.

5. - (1961) - Catálogo Esp. de la Prov. de Barcelona: I Invertebrados, pp. 29-47. Barcelona.

6. Lagar, A. (1954) - Los Bathysciitae de la Provincia de Barcelona. Speleon, año V, no 4, pp. 247-259.

7. DIEGK (1869) - Berl. ent.Zs. XIII, p. 350.

8. Zariquier, R. (1922) - Bathysciinae catalanes (Col Silphidae). But. Inst. Cat. Hist. Nat. XXII, p. 162, pl. XI.

9. - (1924) - Contribución al estudio de los Bathysciinae catalanes. Treb. Mus. Cien. Nat. Barcelona, IV, no 7, pp. 10-12, fig. 3, 4, 6.

10. Llopis, N. (1947) - Contribución al conocimiento de la Morfoestructura de los Catalánides. C.S.I.C., 372 pag., 305 lam. Barcelona.

11. Renault, Ph. (1961) - Sur les caractères des fentes de décollement. Ann. de Spél., T. VI, fasc. 1, Moulis. 


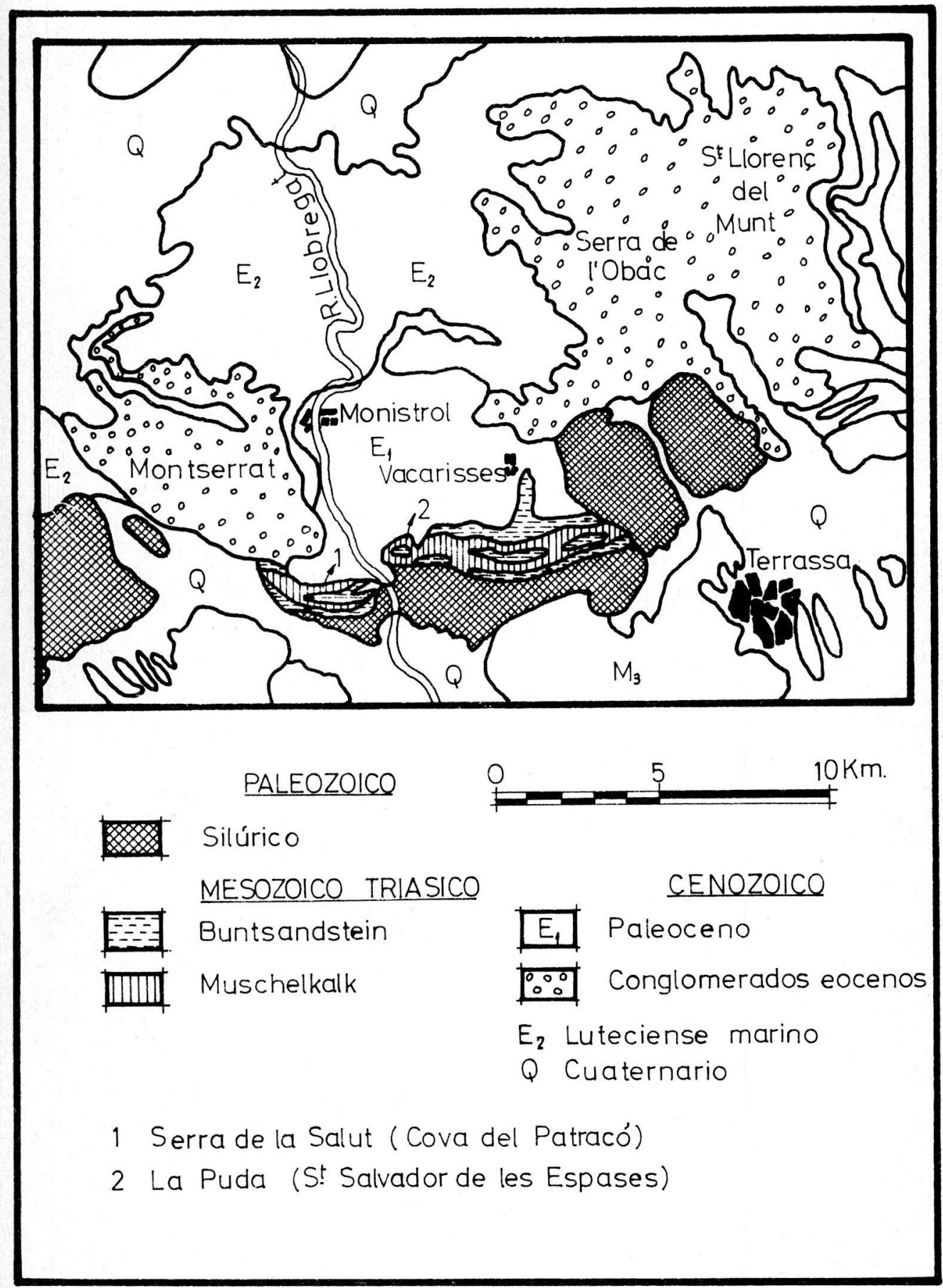

